\title{
ENHANCEMENT OF ENERGY EFFICIENCY AND THROUGHPUT USING CSMA/CA DCF OPERATION FOR CIM
}

\author{
Puneeth Naik. S $\mathbf{S}^{1}$ JayanthiK.Murthy ${ }^{2}$ \\ ${ }^{1}$ MTech-Student (Digital communications), Dept. of Electronics and communication, BMSCE, Bangalore Karnataka- \\ 2013, India \\ ${ }^{2}$ Associate professor (PG Studies), Dept. of Electronics and communication, BMSCE, Bangalore Karnataka-2013, India
}

\begin{abstract}
Advancements in wireless sensor network technology (WSN) have lead researchers to work on wide variety of distributed sensing applications. They have better potential to work without human attention after deployment in large physical environments. Out of all large physical environments that sensing is dealt with, Critical infrastructure monitoring (CIM) emerges as a prominent application. The Major requirements of CIM are reliable data transfer and long deployment life time. These networks are also expected to operate in extremely low-cost communication environment. In this paper we propose a DCF integrated MAC (Medium access control) protocol to cater CIM with better throughput. We have used an infrastructure based wireless sensor network in association with back off algorithm. The simulation shows that DCF integrated channel access method would offer better throughput for CIM which happens to be one of the major requirement.
\end{abstract}

Keywords: CIM (Critical infrastructure monitoring), WSN (Wireless sensor networks), MAC (Medium Access control), DCF (Distributed Coordination function). CS (Carrier sensing).

-****

\section{INTRODUCTION}

The Advantages and advancements of wireless communication, networking and data processing techniques have increased the potential of wireless sensor networks. These sensor networks are robust enough to sense large physical environments, process the data and route the same to the desired destination. The applications of these sensor networks have been consistently increasing and major one's to name are i) Disaster monitoring, ii)Surveillance, iii) Target tracing and iv) Health monitoring [1].

When we review the large physical environments closely, we see that critical infrastructure (CI) have received less attention in terms of monitoring prospective.

Critical Infrastructures: Critical infrastructures are defines as large physical environments which can run up to kilometers. They are very much essential for better functioning of society and economy. Just to list a few, i) Oil and Gas pipeline monitoring, ii) Gas material detection, iii) Water leakage detection. Predominantly CI's are monitored using the traditional techniques. Traditional techniques demands high human attention more frequently and are not much reliable.Monitoring CI's would help in ensuring prevention, maintenance, safety and reliability. It also helps in prevention of catastrophic failures, damages due to leakages (Oil, Gas)[2]. The same has been shown in the figure 1

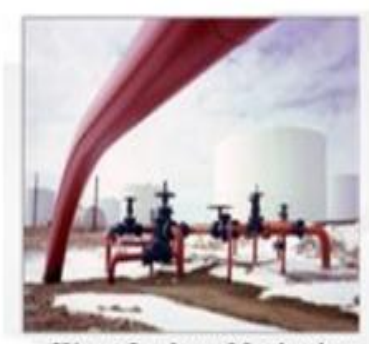

Water Leakage Monitoring

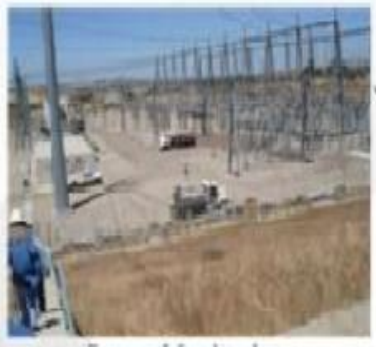

Pemer Menitering
Fig 1: Water leakage Monitoring, Power Monitoring

While Monitoring CI's sensors would produce large amount of data which can be basically categorized into 1) Normal Data 2) Emergency data. These sensed data needs to be transmitted reliably. In wireless sensor networks channel access and energy of the nodes playa important role as all the sensor nodes in the network needs to be treated in fair manner. Improper assignment of channels would affect the throughput, and leads to inefficient utilization of energy [3].

The most suitable and challenging area in networking to work with is MAC Protocols. MAC basically works with channels and helps in achieving the desired throughput and manages the delay. In this article we first describe Distributed coordination function (DCF) concept and its operation. We integrate this concept with MAC protocol in order to enhance the 
throughput for CI's using proper channel management, after which we simulate the same and analyze the results.

\section{RELATED WORK}

The handsome amount of research has been done by authors in [4] on resources management in wireless sensor networks. The IEEE 802.15.4e based CIM MAC which utilizes the time slotted channel hopping concept. The authors in [5] have investigated on the power consumption at end points. They have proposed a low-energy link access for CIM by distributing the access load on the slotted link. The author in [6] has proposed "Low Energy MAC Proposal for TG4kWSNIRI" based on Non Beacon mode, CSMA/CA and ACK. Here the coordinator keeps listening to the channel and the endpoints keep sleeping. If endpoint has data to transmit, sends Data Request frame by using CSMA/CA, and waits for payload frame from Coordinator, failing which the retransmission of data request frame takes place. If the coordinator wants to send a Multicast or broadcast frame then sends a sequence of wakeup calls to make an appointment about the desired frame transmission time. If it has a unicast frame to a particular end point, theit broadcasts the wakeup call and waits for the ACK from the intended receiver failing which resends the wakeup frame. The authors [7] have proposed an IEEE 802.15.4e based CIM MAC protocol which consumes less energy and they do not consider the concept of Coordinator. It uses the idea of batch transmission and coordinated sleeping. In this concept the technique of buffer management at the receiver plays a important role and also uses the ACK concept. This concept y helps in conserving the energy, However it difficult to implement the concept of buffer in all the nodes. In this paper we have considered few inputs from the literature referred above and have worked on throughput and energy aspects of CIM

\section{THROUGHPUT AND ENERGY ATTRIBUTES}

\section{OF WSN}

Major sources of energy waste in wireless sensor network are basically of four types [8] [9].

(i) Collision: The first one is the collision. When a transmitted packet is corrupted due to interference, it has to be discarded and the follow on Retransmissions increase energy consumption. Collision increases latency also.

(ii) Overhearing: The second is overhearing, meaning that a node picks up packets that are destined to other nodes.

(iii) Packet Overhead: The third source is control packet overhead. Sending and receiving control packets consumes energy too and less useful data packets can be transmitted.

(iv) Idle listening: The last major source of inefficiency is idle listening i.e., listening to receive possible traffic that is not sent. This is especially true in many sensor network applications. If nothing is sensed, the sensor node will be in idle state for most of the time.
The main goal of any MAC protocol for sensor network is to minimize the energy waste due to idle listening, overhearing and collision.

\section{MEDIUM ACCESS CONTROL (MAC)}

MAC protocol is responsible for reliable, error free data transfer with minimum retransmissions. The MAC also caters to meet performance requirements such as controlling bandwidth, power awareness, contention resolution, minimize interference and collision avoidance. Data collection in WSN tends to suffer from heavy congestion which basically affects the throughput and the energy consumption in the network.[10] MAC protocols can be categorized as Contention-free and Contention-based, as shown in figure 2.

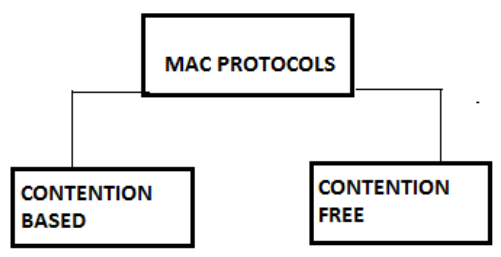

Fig 2: Classification of MAC protocols.

Contention free Protocols: The contention free protocols do not make the assumption that network traffic is intrinsically random, instead traffic is ordered in a bounded channel assignment. These schemes are generally based on TDMA, FDMA or CDMA that utilizes the synchronization technique and the channel access mechanism of the physical layer. However, these schemes are more complex, specialized sensor hardware and there is a dependency on the physical layer [11].

Contention Based Protocols: The contention based protocols use Carrier Sense Multiple Access (CSMA) scheme, where for a node to transmit, it must sense the medium to determine if another node is transmitting. If the medium is busy, the node will defer until the end of the current transmission. After deferral or just before attempting to transmit again, the node shall select a random back-off interval and shall decrement the back-off interval counter while the medium is idle [11].

The MAC sub layer of the IEEE 802.11 defines the Distributed Coordination Function (DCF), the Point Coordination Function (PCF), and the Hybrid Coordination Function (HCF). The focus will be on the DCF that allows automatic medium sharing [12].

\section{DISTRIBUTED COORDINATION FUNCTION}

\section{(DCF)}

The basic access mechanism called DCF is a carrier senses multiple access collision avoidance (CSMA/CA) mechanism. The CSMA protocol allows a station wishing to transmit to sense the medium, if the medium is busy it defer its 
transmission but if the medium is free then the station is allowed to transmit. CSMA is very effective when the medium does not have high traffic, since all medium transmit with minimum delay. Stations transmitting at the same time result in collision as the protocol initially are designed for single channel transmission. CA allows the medium that is busy and defers to wait and allow the medium to be free for a specific time called distributed inter frame space (DIFS) then the station is allowed to transmit.[13]

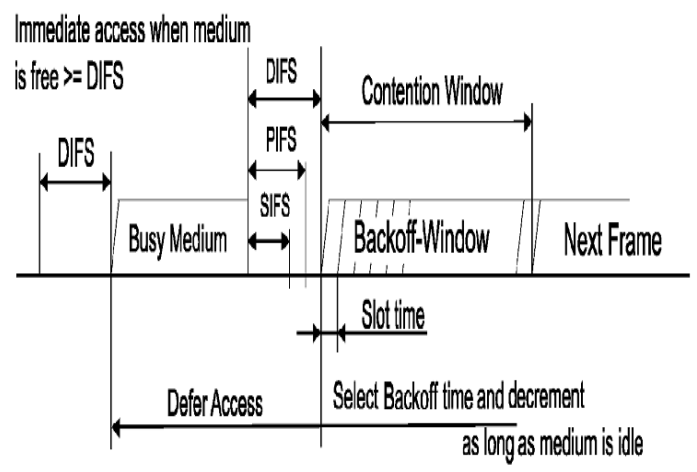

Fig 3: DCF Operation.

Multiple collision occur more frequently after a busy period when there are multiple stations waiting on the medium to transmit their data. This situation necessitates a random backoff procedure to resolve medium contention conflicts through carrier sense (CS) functions [13, 14].

The IEEE 802.11 DCF protocol works in two different modes 1) infrastructure mode and 2)Ad hoc mode. In infrastructure mode communication between modes must go through a central node called coordinator node, while in Ad hoc mode nodes communicate directly with each other. Infrastructure mode fits for static large infrastructure applications $[15,16]$. In this paper we focus on communication which is based on infrastructure mode and we utilize IEEE 802.11 DCF infrastructure mode along with MAC protocol for the CIM. By enhancing throughput and maintaining channel access fairness within the network, the quality of service is enhanced and consequently the WSN information assurance is supported for CIM.

\section{COMMUNICATION PROCESS}

The main motto of the CIM is to facilitate single point to multi-thousands of point's Communication for critical infrastructure monitoring with low energy. The main components of CIM network are coordinator or collector, devices or end points, and repeater. Coordinator is generally main powered, and endpoints are battery powered. Topology used in CIM is star topology that supports one to multipoint communication. CIM network consists of only one coordinator that supports many devices. The present 802.15.1, Bluetooth has a short range and cannot support thousands of endpoints due to low capacity. The IEEE 802.15.3 also has a short range and aimed high data rate applications. IEEE 802.15.4/4e MACs supports low data rate applications. However, they need modifications to support such a large network with very lossy channel. IEEE 802.15.4e supports mainly TDMA approach which cannot work well for CIM networks due to large number of endpoints as it leads to lot of delay.

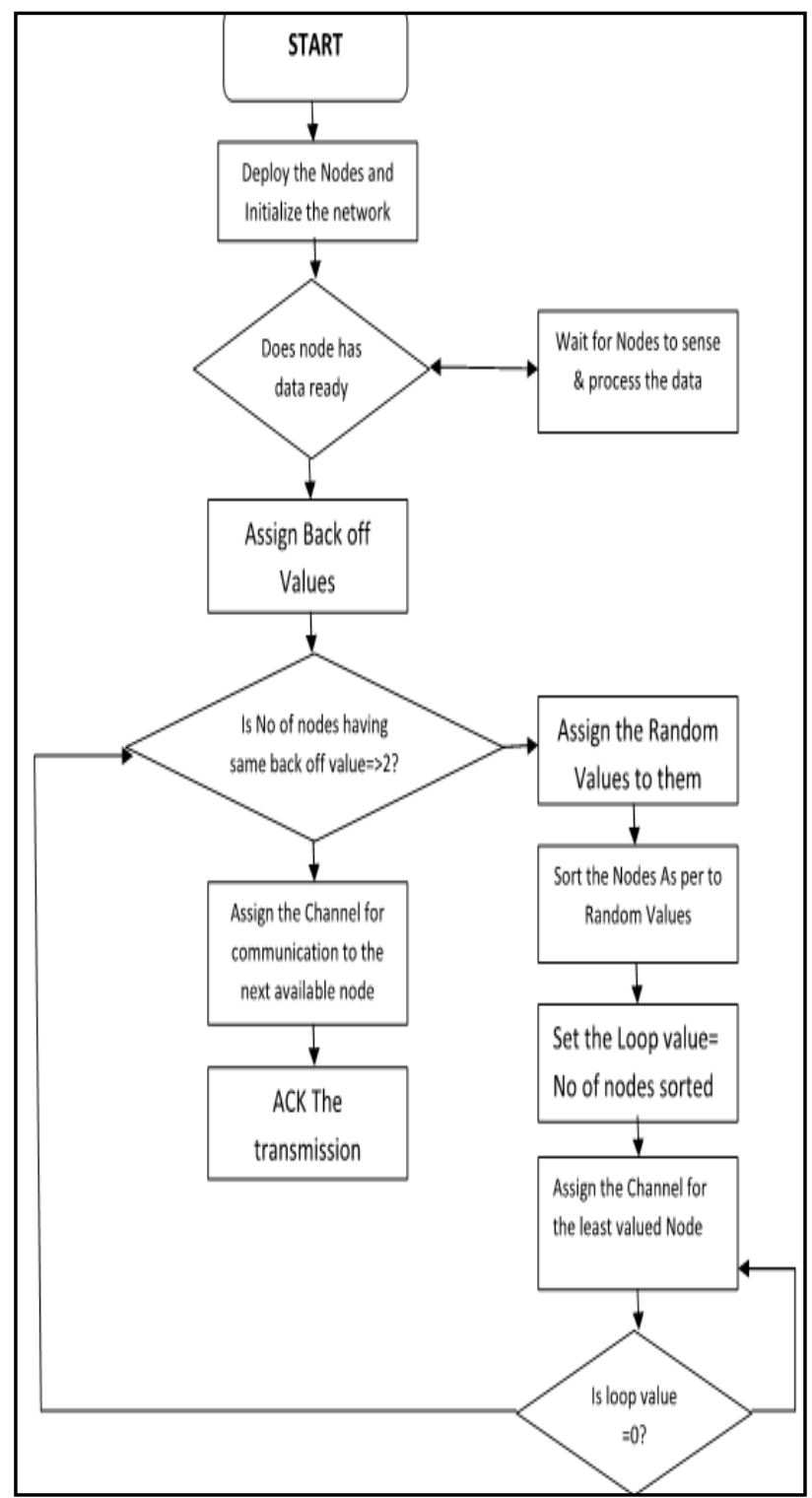

Flow Chart 1: Communication Process

Unlike traditional MAC protocols in this project we consider a unique approach to satisfy the CIM demands. A IEEE 802.11 based MAC protocol and the concept of DCF are used. The performance analysis is done based on the analysis of the channel assignment to the endpoints and the throughput. 


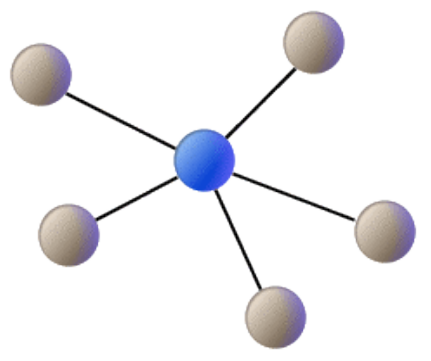

Fig 4: Star topology

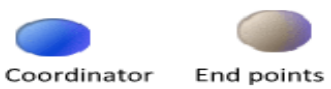

\section{PERFORMANCE ANALYSIS}

As we are dealing with infrastructure based wireless sensor network, the coordinator dominates all the end points and manages radio resources and power. When we consider uplink communication i.e. from end pints to the coordinator, channel access management needs to be handled by MAC protocol. In this implementation we have considered the DCF operation with standard IEEE 802.11 MAC protocol. Initially when we deploy the nodes and initialize the network, we assume that all these nodes have the monitored data with them and they are ready to contend for the channel. The implementation of the same has been shown in the figure

Now the MAC protocol with DCF operation would allocate the backoff values to all the nodes which are deployed. Once the nodes have the backoff value assigned, they start listening to the channel and executes the CSMA/CA technique. If the channel is busy it waits for a time called DIFS and still if it hears the channel to be busy then it will backoff again. The value of the back off counter would be reduced by 1 on every successful transmission on the channel. Likewise all the deployed nodes would be given with a particular frame for transmission of its sensed data in a fair manner.

Special Case: If the back off values of two or more nodes $n$ the network becomes zero at the same time, then all the nodes with same back off value would start there transmissions. This situation would lead to losing a complete frame length allocated to these nodes. To avoid this scenario, we have included an additional algorithm in this implementation to handle the case efficiently.

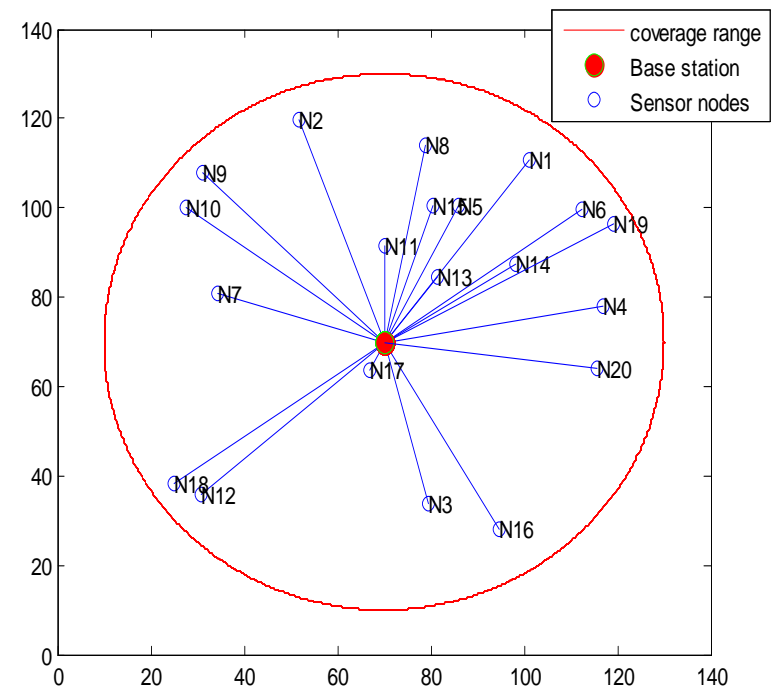

Fig 5: Network Deployment of CIM

When two nodes are assigned with the same back off values and becomes zero (Upon CSMA), MAC protocol assigns another pair of random values to them. They nodes would be sorted in ascending order and the one with the least random value will be given with a chance to transmit the information over the channel.

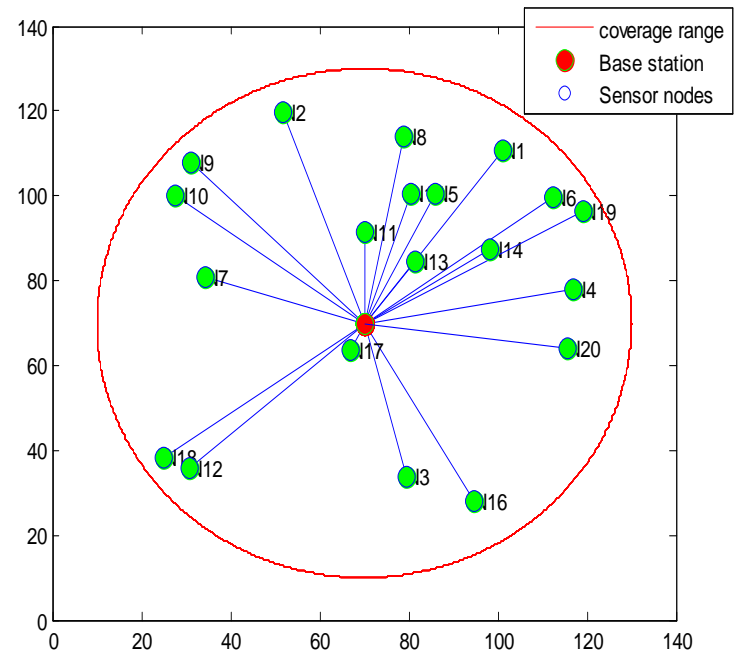

Fig 6: successful assignment of channels.

The process will be repeated until all the nodes which had the same back off value are given with an opportunity to transmit the information. With this technique we were able to avoid collisions and enhance the throughput, avoid energy consumption and reduce the latency. 
The Parameters that we had considered for the implementation are as follows

\begin{tabular}{|l|l|l|}
\hline SI NO & PARAMETER NAME & VALUES \\
\hline 1 & $\begin{array}{l}\text { Number of Sensor } \\
\text { nodes }\end{array}$ & $\begin{array}{l}\text { 20(Random } \\
\text { Deployment) }\end{array}$ \\
\hline 2 & SIFS & $0.01 \mathrm{sec}$ \\
\hline 3 & DIFS & $0.05 \mathrm{sec}$ \\
\hline 4 & Physical Area & $140 \mathrm{~m} \mathrm{x} \mathrm{140m}$ \\
\hline 5 & Frame length & 10 \\
\hline 6 & Back off range & 0 to $20($ Random) \\
\hline 7 & frequency & $2.4 \mathrm{GHz}$ \\
\hline 8 & Threshold Noise & $0.7(\mathrm{Random})$ \\
\hline
\end{tabular}

\section{RESULTS AND DISCUSSIONS}

We found that the throughput has been increased as per the results plotted using the simulation values. We also see that the network congestion has been intelligently handled by using the DCF operation. All the nodes in the deployed physical area are given with a chance in the fair manner. The frames are also been utilized efficiently. The frame drops have been reduced which basically happens due to inappropriate planning of channel allocation. The drops which are shown in plot are based on the noise parameter which we have assumed and not because of the failing in handling the channel access. Using the proposed technique we can aid the CIM in a efficient and reliable approach. The plot of the same has been shown in figure 7

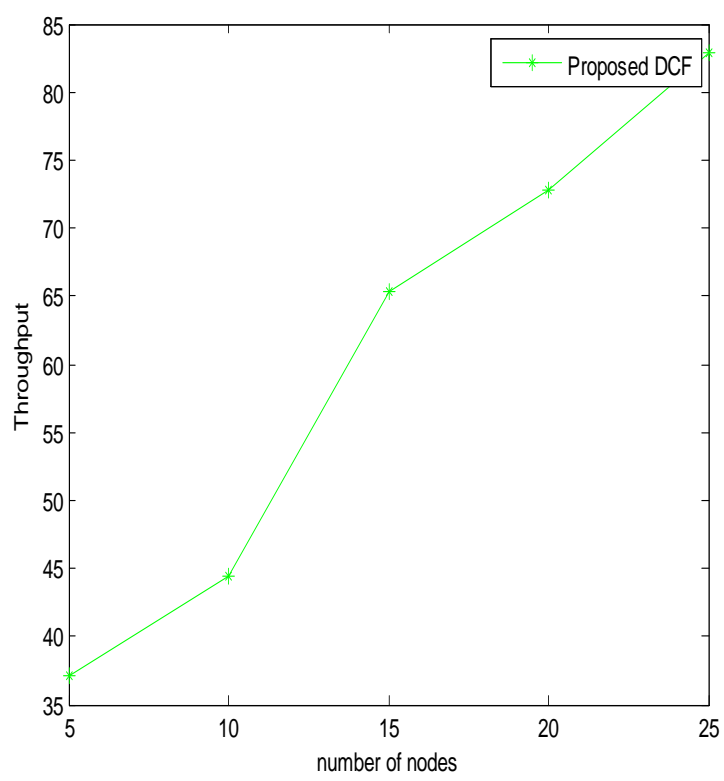

Fig 7: Plot of throughput

\section{CONCLUSIONS AND FUTURE WORK}

We proposed a IEEE 802.11 based MAC protocol integrated with CAMS/CA based DCF technique for CIM. We have used a infrastructure based wireless sensor network which helps the end points to have a minimal work assigned and the coordinator which is main powered with the maximum work . This prolongs the deployment life time of end points as they are battery driven. We have showed the better throughput by handling the channel access using CSMA/CA DCF operation. The proposed protocol is simple implement and flexible in terms of network size. In future we will extend our work to include slotted aloha protocol in this implementation and do the performance analysis in aiding CIM.

\section{REFERENCES}

[1]. S. Hayat, N. Javaid, Z. A. Khan, A. Shareef, A. Mahmood, S. H. Bouk, "Energy Efficient MAC Protocols" Http://arxiv.org/abs/1207.2567v1

[2]. Niamat Ullah, M. SanaullahChowdhury, Mosaddique Al Ameen, andKyung SupKwak, "Energy Efficient MAC Protocol for Low-Energy Critical Infrastructure Monitoring Networks Using Wakeup Radio" International Journal of Distributed Sensor Networks Volume 2012, Article ID 504946,15 pages doi:10.1155/2012/504946

[3]. J. Becker, M. Calcagno, and D. Howard, "Low Energy Critical Infrastructure Monitoring, doc.: IEEE 802.15-100053-00-wng0-,"

2010 ,

https://mentor.ieee.org/802.15/documents.

[4]. Schwoerer and N. Dejean, "Elster \& France Telecom proposal", doc.: IEEE 802.15-11-0479-01-004k, 2011, https://mentor.ieee.org/802.15/documents

[5]. S.S.Joo, J.A.Jun, and C.S.Pyo, "MAC Proposal for LowEnergy Wide Area Monitoring, doc.: IEEE802.15-11-059701-004k," 2011, https://mentor.ieee.org/802.15/documents

[6]. Y. Yang et al., "Low Energy MAC Proposal for TG4kWSNIRI, doc.: IEEE 802.15-11-0596-00-004k," 2011, https://mentor.ieee.org/802.15/documents.

[7]. H. Liu, G. Yao, and P. Xu, "Energy Efficient MAC protocol for Low Energy Critical Infrastructure Networks" Doc:

IEEE802.15-11-0619-01-004k-2011, hhts://mentor.ieee.org.

[8]. Wei Ye, J.Heidemann and D. Estrin: An Energy-Efficient MAC Protocol for Wireless Sensor Networks, IEEE INFOCOM, New York, Vol. 2, pp. 1567-1576 (June 2002).

[9]. Tijs van Dam, Koen Langendoen: An Adaptive Energy Efficient MAC Protocol for Wireless Networks, in Proceedings of the First ACM Conference on Embedded Networked Sensor Systems (November 2003)v

[10]. Kumar, S. Raghavan, V. Deng, Medium Access Control protocols for ad hoc wireless networks: A survey. Ad Hoc Networks 4, 2006, pp. 326-358.

[11]. Urmila A. Patil, Smita V. Modi, Suma B.J. "Analysis and Implementation of IEEE 802.11 MAC Protocol for Wireless Sensor Networks "International Journal of 
Engineering Science and Innovative Technology (IJESIT) Volume 2, Issue 5, September 2013.

[12]. V. Vitsas, P. Chatzimisios, A. C. Boucouvalas, P. Raptis, K. Paparrizos and D. Kleftouris, "Enhancing performance of the IEEE 802.11 Distributed Coordination Function via packet bursting" IEEE Communications Society Globecom 2004 Workshops 0-7803-87 98-8/04/\$20.00 @2004 IEEE

[13]. J. Polastre, J. Hill, and D. Culler, "Versatile low power media access for wireless sensor networks," in Proceedings of the $2^{\text {nd }}$ International Conference on Embedded Networked Sensor Systems (SenSys '04), pp. 95-107, Baltimore, Md, USA, November2004.

[14]. J.Lopez, J.A.Montenegro, and R.Roman, "Service oriented security architecture for CII based on sensor networks," in 2nd International Workshop on Security, Privacy and Trust in Pervasive and Ubiquitous Computing (SecPerU '06), pp. 1-6, June 2006.

[15]. M. Albano, S. Chessa, and R. Di Pietro, "A model with applications for data survivability in Critical Infrastructures, ” International Journal of Information Assurance and Security, vol. 4, pp. 629-639, 2009.

[16]. B. Rolfe," Preliminary Fragmentation Proposal for TG4k, doc. IEEE 802.15-11-0478-00-004k,"2011, https://mentor.ieee.org/802.15/documents 